\title{
Antibacterial efficacy of core-shell nanostructures encapsulating gentamicin against an in vivo intracellular Salmonella model
}

This article was published in the following Dove Press journal:

International Journal of Nanomedicine

I December 2009

Number of times this article has been viewed

\author{
Ashish Ranjan' \\ Nikorn Pothayee ${ }^{2,3}$ \\ Mohammed N Seleem ${ }^{2}$ \\ Ronald D Tyler J $\mathrm{r}^{4}$ \\ Bonnie Brenseke ${ }^{4}$ \\ Nammalwar Sriranganathan ${ }^{2,4}$ \\ Judy S Riffle ${ }^{2,3}$ \\ Ramanathan Kasimanickam' \\ 'Department of Large Animal Clinical \\ Sciences, ${ }^{2}$ Institute for Critical \\ Technology and Applied Science, \\ ${ }^{3}$ Macromolecules and Interfaces \\ Institute, ${ }^{4}$ Department of Biomedical \\ Sciences and Pathobiology, Virginia \\ Polytechnic Institute and State \\ University, Blacksburg, VA
}

Correspondence: Nammalwar

Sriranganathan

Department of Biomedical Sciences and

Pathobiology, Virginia Polytechnic Institute and State University Blacksburg VA, USA

Tel + I 540 23 | 7|7|

Email nathans@vt.edu

\begin{abstract}
Pluronic based core-shell nanostructures encapsulating gentamicin were designed in this study. Block copolymers of (PAA ${ }^{-+} \mathrm{Na}-b$-(PEO- $b$-PPO- $b$-PEO)-b-PAA ${ }^{-+} \mathrm{Na}$ ) were blended with $\mathrm{PAA}^{-} \mathrm{Na}^{+}$and complexed with the polycationic antibiotic gentamicin to form nanostructures. Synthesized nanostructures had a hydrodynamic diameter of $210 \mathrm{~nm}$, zeta potentials of $-0.7( \pm 0.2)$, and incorporated $~ 20 \%$ by weight of gentamicin. Nanostructures upon co-incubation with J774A.1 macrophage cells showed no adverse toxicity in vitro. Nanostructures administered in vivo either at multiple dosage of $5 \mu \mathrm{g} \mathrm{g}^{-1}$ or single dosage of $15 \mu \mathrm{g} \mathrm{g}^{-1}$ in AJ-646 mice infected with Salmonella resulted in significant reduction of viable bacteria in the liver and spleen. Histopathological evaluation for concentration-dependent toxicity at a dosage of $15 \mu \mathrm{g} \mathrm{g}^{-1}$ revealed mineralized deposits in 50\% kidney tissues of free gentamicin-treated mice which in contrast was absent in nanostructure-treated mice. Thus, encapsulation of gentamicin in nanostructures may reduce toxicity and improve in vivo bacterial clearance.
\end{abstract}

Keywords: gentamicin, core-shell nanostructures, Salmonella

\section{Introduction}

Delivery systems capable of transferring cell-impermeable drugs into cells have tremendous potential for improving therapeutic efficacies in vivo. ${ }^{1}$ In the last few decades, nanotechnology has been increasingly employed in new drug delivery systems (DDS). DDS developed through nanotechnology may allow for reductions in dosage and dosage frequency and may also prevent toxicity associated with conventional therapy. This may be especially beneficial for chronic intracellular infections which now require long-duration combination therapies. ${ }^{2}$ For example, aminoglycosides are a common class of antibiotics utilized clinically for treating bacterial infections. They have broad activities against both Gram-negative and Gram-positive bacteria. ${ }^{3}$ However, the action of aminoglycosides is concentration-dependent and free gentamicin filters rapidly through the renal system, thus necessitating high dosages. Administration of high dosages of aminoglycosides may result in nephrotoxicity and ototoxicity, which may be prevented with nanomedicine-based therapy. ${ }^{4}$

To achieve intracellular delivery of aminoglycosides, several drug delivery strategies have been utilized including liposomes and polymeric carriers. ${ }^{5,6}$ These drug delivery systems have improved the intracellular delivery of aminoglycosides, but suffer from low drug incorporation. Core-shell drug delivery systems have recently attracted considerable attention due to their unique ability in encapsulating charged therapeutic molecules. ${ }^{2}$ The unique ability is conferred to the core-shell structures 
mainly by fabricating the core to contain high amounts of ionic species and tailoring shell with specific hydrophobic/ hydrophilic chemistry as shown schematically in Figure 1. This may result in encapsulation of high payload of oppositely charged ionic species like gentamicin inside the cores and successful interaction of the shell's hydrophobic segment with the cell membranes.

We previously reported synthesis of core-shell nanostructure with amphiphilic shell containing pluronic F68 which can incorporate up to $\sim 25 \%$ by weight of gentamicin and their in vitro trafficking and efficacy in infected macrophage cells. ${ }^{22}$ Pluronics have widely been used as excipients in pharmaceutics. $^{7}$ Pluronic ${ }^{\mathrm{TM}}$ triblock copolymers are comprised of hydrophilic poly(ethylene oxide) (PEO) terminal blocks with a hydrophobic poly(propylene oxide) (PPO) central block (ie, PEO- $b$-PPO- $b$-PEO) in a basic A-B-A structure: $\mathrm{EO}_{\mathrm{x} / 2}{ }^{-}$ $\mathrm{PO}_{\mathrm{y}}-\mathrm{EO}_{\mathrm{x} / 2}{ }^{8}$ Different pluronic copolymers are characterized distinctly by their hydrophilic-lipophilic balance (HLB). For example, the HLB of pluronic F68 is 29 whereas that of pluronic P85 is 16. Therefore, pluronic F68 is relatively less hydrophobic than pluronic P85. Pluronics have been investigated extensively for cancer chemotherapy of multidrug resistant (MDR) tumors. ${ }^{9}$ By varying the lengths of the hydrophobic propylene oxide (y) and hydrophilic ethylene oxide (x) segments, increased potency and intracellular uptake of the DDS were seen in the tumor cells. ${ }^{10}$ Studies on $\mathrm{KBv}$ cell lines have shown that the accumulation of pluronics inside the cells is dependent on HLB. In general, pluronics with HLB of 20-29 had minimal effect on drug transport. In contrast, pluronics with HLB less than 20 exhibited varying degree of activity against MDR cells. ${ }^{8}$ Thus, the triblock arrangement provides an advantage of altering the hydrophilic/hydrophobic balance to suit a particular therapeutic application. ${ }^{11}$ Therefore, its incorporation in DDS for cell specific therapy may be highly favorable, ${ }^{12}$ especially for intracellular infections.

Preclinical studies conducted previously with biodegradable DDS made from a combination of poly( $\varepsilon$-caprolactone) (PCL) and Pluronic F68 ${ }^{\mathrm{TM}}$ did not cause any adverse toxicological response in rats or dogs. ${ }^{18}$ In this study, we designed coreshell nanostructure by synthesizing $\mathrm{PAA}^{-+} \mathrm{Na}-b-\mathrm{PEO}-b$-PPO$b$-PEO-b-PAA ${ }^{-+} \mathrm{Na}$ block copolymers and complexed them with gentamicin to form nanostructures with PEO- $b$-PPO- $b$ PEO shells, and PAA cores containing the antibiotic shown schematically in Figure 1. The efficacies of these core-shell nanostructures were investigated in an in vivo intracellular

\section{Anionic block copolymers}

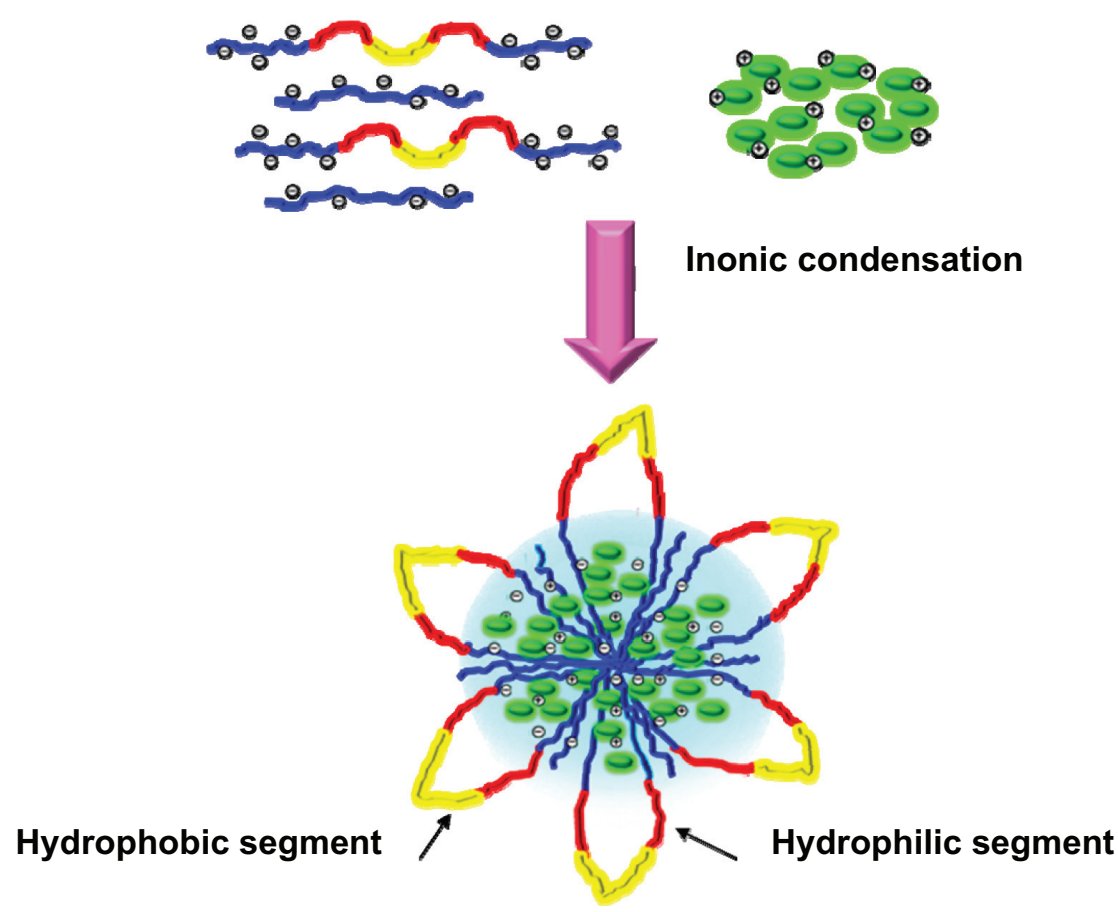

Amphiphilic core-shell complexes

\section{Aminoglycosides}

Inonic condensation

Figure I Schematic illustration of methodology for preparing core-shell nanostructure encapsulating gentamicin. 
Salmonella mouse infection model. Our objectives also included an evaluation of the observed reduction in gentamicin toxicity that resulted from encapsulation in the cores.

\section{Materials and methods Materials}

All chemicals were purchased from Sigma-Aldrich unless otherwise noted. $t$-Butyl acrylate (tBA) was distilled from calcium hydride under vacuum prior to polymerization. Pluronic F68 $\left(\mathrm{M}_{\mathrm{n}} \sim 8,300 \mathrm{~g} \mathrm{~mol}^{-1}\right)$ was kindly provided by BASF (NJ, USA). Pluronic F68 and polyethylene oxide monomethyl ether $\left(M_{n} \sim 2,000 \mathrm{~g} \mathrm{~mol}^{-1}\right)$ were dried under vacuum at $70^{\circ} \mathrm{C}$ for 48 hours prior to any polymerizations.

\section{Preparation of core-shell nanostructures loaded with gentamicin}

Block copolymers of poly(sodium acrylate)-(PEO- $b$-PPO$b$-PEO)-poly(sodium acrylate) (PAA ${ }^{-+} \mathrm{Na}-(\mathrm{PEO}-b$-PPO$b$-PEO)-PAA $\left.{ }^{-+} \mathrm{Na}\right)$ and $\mathrm{PEO}-\mathrm{PAA}^{-+} \mathrm{Na}$ were prepared according to a previously published procedure. ${ }^{13}$ Briefly, PEO- $b$-PPO- $b$-PEO (Pluronic F68) with hydroxyl terminal groups was derivatized with 2-bromoisobutyryl bromide to provide bromoalkyl functional endgroups and then t-butyl acrylate was polymerized by controlled atom transfer radical polymerization (ATRP). The protective t-butyl groups were subsequently removed with trifluoroacetic acid and the polymer was neutralized with $\mathrm{NaOH}$ to form the sodium salt $\left(\mathrm{PAA}^{-+} \mathrm{Na}-\left(\mathrm{PEO}-b\right.\right.$-PPO- $b$-PEO)-PAA $\left.{ }^{-+} \mathrm{Na}\right)$. In a $100-\mathrm{mL}$ round-bottom flask, $34 \mathrm{mg}$ of $\mathrm{PAA}^{-+} \mathrm{Na}-(\mathrm{PEO}-$ $b$-PPO- $b$-PEO)-PAA ${ }^{-+} \mathrm{Na}\left(1.90 \times 10^{-4}\right.$ eq of anions $)$ and $16 \mathrm{mg}$ of $\mathrm{PAA}^{-+} \mathrm{Na}\left(1.6 \times 10^{-4}\right.$ eq of anions $)$ were dissolved in $50 \mathrm{~mL}$ of DI water and the $\mathrm{pH}$ was adjusted to 7 with $\mathrm{NaOH}(1 \mathrm{~N})$. The solution was placed in a sonication bath and $5 \mathrm{~mL}$ of gentamicin sulfate solution $\left(10 \mathrm{mg} \mathrm{mL}^{-1}\right.$ gentamicin sulfate, equal to $30 \mathrm{mg}$ gentamicin, $3.5 \times 10^{-4}$ eq of cations) was added via syringe to form a turbid dispersion. Any non-complexed free gentamicin sulfate was removed by dialysis against $4 \mathrm{~L}$ of deionized (DI) water at $4^{\circ} \mathrm{C}$ for 24 hours and the core-shell nanostructures were recovered by freeze-drying. Core-shell nanostructure prepared by this methodology was later utilized for in vivo treatment efficacy studies at dosage of $5 \mu \mathrm{g} \mathrm{g}^{-1}$ and $15 \mu \mathrm{g} \mathrm{g}^{-1}$ respectively. The nomenclature describing the mice treated at $5 \mu \mathrm{g} \mathrm{g}^{-1}$ adopted for this paper is D1 (D1N for the group treated with coreshell nanostructure and D1G for the group treated with free gentamicin alone). Similarly, the mice administered with $15 \mu \mathrm{g}$ are designated as D2 (D2N for the group treated with the core-shell nanostructure and D2G for mice group treated with free gentamicin alone).

\section{Characterization of complexes}

The solute sizes and zeta potentials of the complexes were characterized by DLS with a Zetasizer 1000 HS with laser diffractometry (Malvern Instruments, Malvern, UK) at a scattering angle of $90^{\circ}$. Each complex (1 mg) was dispersed in $1 \mathrm{~mL}$ of DI water and analyzed. Measurements were done in triplicate for each batch of particles. The results were taken as the average of three measurements.

\section{Gentamicin concentration in the nanostructure}

The amount of gentamicin loaded into the core-shell nanostructures was determined via an $o$-phthalaldehyde assay according to a previously published procedure. ${ }^{14}$ To quantify the amount of encapsulated gentamicin, the complexes were dissolved in borate buffer at $\mathrm{pH} 9.7$ to disintegrate the particles and release the drug in the free amine form. The primary amine on gentamicin was then reacted with an excess of phthalaldehyde in the presence of mercaptoethanol to produce a derivative that was quantified via UV-visible spectroscopy. For derivatization, gentamicin solution, phthalaldehyde reagent containing mercaptoethanol, and isopropanol (to avoid precipitation of the products) were reacted for 30 minutes at room temperature, and the absorbance of the product was measured at $335 \mathrm{~nm}$.

\section{In vitro MTS toxicity assessment}

An in vitro homogeneous, colorimetric CellTiter $96^{\circledR}$ AQueous Non-Radioactive Cell Proliferation MTS (3-(4,5dimethylthiazol-2-yl)-5-(3-carboxymethoxyphenyl)-2-(4sulfophenyl)-2H-tetrazolium) Assay (Promega Corporation) for determining the numbers of viable J774A.1 cells was utilized to determine any cytotoxicity of the polymers and the nanostructures. Briefly, $\sim 2 \times 10^{4}$ J774A. 1 cells suspended in $200 \mu \mathrm{L}$ of DMEM supplemented with $10 \%$ fetal bovine serum (FBS), L-glutamine, $\mathrm{NaHCO}_{3}$, pyridoxine- $\mathrm{HCl}$, and $4.5 \%$ glucose and preserved with $1 \%$ penicillin-streptomycin solution were seeded in 96-well plates and incubated for 24 hours at $37^{\circ} \mathrm{C}$ in a $5 \% \mathrm{CO}_{2}$ atmosphere. The J774A. 1 cells were further incubated with $250 \mu \mathrm{g} / \mathrm{mL}$ of free gentamicin, copolymer, core-shell nanostructures encapsulating gentamicin along with the appropriate untreated control for 24 hours. The culture media was discarded, and the cells in each well were washed with PBS and re-suspended with $100 \mu \mathrm{L}$ of cell culture media. Then $20 \mu \mathrm{L}$ of CellTiter 
$96^{\circledR}$ AQueous reagent solution was pipetted into each well, and the plates were incubated for 4 hours at $37^{\circ} \mathrm{C}$ in a humidified $5 \% \mathrm{CO}_{2}$ atmosphere. The absorbance at $490 \mathrm{~nm}$ was recorded using a 96-well Elisa plate reader (SoftMax Pro Inc., USA). Results were expressed as the percentage mean absorbance by cells upon incubation with various treatments (nanostructure, copolymer or free gentamicin) with respect to incubation in untreated control.

\section{In vivo toxicity}

To assess concentration/dose dependent toxicity, kidney tissue from the untreated, D2N- or D2G-treated mice were harvested and assessed for histopathological changes. The kidneys were fixed in 10\% neutral buffered formalin, routinely processed into paraffin blocks, and 5 micron sections were stained with hematoxylin and eosin on glass slides (Virginia-Maryland Regional College of Veterinary Medicine, Veterinary Teaching Hospital). Tissue samples were examined independently by light microscopy and scored by two veterinary pathologists. The pathologists were blinded to obtain unbiased assessment. The kidneys were scored on a scale of 0 to 4 based on the degree of inflammation as reported before. ${ }^{15,16}$ Briefly, a semi-quantitative scale of change consisting of whole numbers with scores from 0 to 4 was given as $(0)=$ unremarkable, $(1)=$ minimum, (2) = mild, (3)= moderate, and (4)= marked. Changes include inflammation (infiltration of inflammatory cells), necrosis (morphologic changes of renal parenchyma cells consistent with degeneration and necrosis) and vascular disruptions (hemorrhage, edema and other signs of vascular leakage). The percentage of affected section of tissue being affected is what was used to score any change viz. $0 \%=$ unremarkable (0), $0 \%$ to $5 \%=$ minimal (1), $6 \%$ to $10 \%=\operatorname{mild}(2), 11 \%$ to $20 \%=$ moderate $(3),>20 \%=$ marked. ${ }^{4}$ Furthermore, any samples with the presence of mineral deposits associated with regions of inflammation were assigned an additional 0.5 points to take mineralization into account for final analysis.

\section{Treatment efficacy of core-shell nanostructures against against \\ S. typhimurium}

S. enterica typhimurium (wild type) was grown overnight in Luria-Bertani (LB) broth, centrifuged, washed in PBS and serially diluted to achieve a final suspension containing 10,000 colony forming units (cfu) per mL. Twenty-five AJ 646 mice ( 6 to 8 weeks old) were injected intraperitoneally (ip) with $100 \mu \mathrm{L}$ of inocula containing $1000 \mathrm{cfu}$ per mouse. After 48 hours of infection, 5 AJ 646 mice per group were treated with either D1G (in PBS) or the D1N ip at a recommended dosage of $5 \mu \mathrm{g} \mathrm{g}^{-1}$ of body weight. ${ }^{17}$ The dosage was repeated twice at intervals of 24 hours, ip for a total of 3 doses. At day 2 (post third ip injection), the mice were euthanized, the spleens and livers were removed aseptically, and the organs were crushed and homogenized for 1 to $2 \mathrm{~min}$ in the presence of $2 \mathrm{~mL}$ of LB broth. Aliquots $(500 \mu \mathrm{L})$ of the organ suspensions were serially diluted in $4.5 \mathrm{~mL}$ of LB broth to a maximum of $10^{5}$-fold dilution. Subsequently, the dilutions were plated on tryptic soy agar plates, incubated overnight at $37^{\circ} \mathrm{C}$, and the resulting grey mucoid, discrete colonies were counted for plates containing between 30 and 300 colonies. Also, two groups of Salmonella-infected mice ( 5 mice per group) were administered with either D2G or the D2N at a single ip dosage of $15 \mu \mathrm{g} \mathrm{g}^{-1}$ of body weight to determine any concentration-dependent toxicity on the kidneys. The mice were euthanized at day 5 of the study along with the normally administered dosed mice.

\section{Statistical analysis}

The mean absorbance in the MTS assay was compared between the groups using analysis of variance. The kidney scores were compared between the three groups using the Kruskal-Wallis Test followed by Dunn's procedure for multiple comparisons. Statistical significance was set to alpha $=0.05$. Analyses were performed using JMP (ANOVA and Kruskal-Wallis test) and SAS (Dunn's procedure for multiple comparisons).

\section{Results}

\section{Core-shell nanostructures}

DLS showed that polymer-gentamicin core-shell nanostructures had a mean intensity average diameter of $210( \pm 17) \mathrm{nm}$ and had negligible zeta potentials, ranging from $-0.7( \pm 0.2) \mathrm{mv}$. The amount of gentamicin encapsulation based on a UV spectroscopic analyis of an $o$-phthalaldehyde-mercaptoethanol derivative of the drug showed an encapsulation of $\sim 25 \%$. The rather high drug concentration of $\sim 25 \%$ by weight is attributed to strong interactions between the cationic gentamicin and the anionic polymer.

\section{In vitro MTS assay}

MTS assays conducted on the free gentamicin, copolymer, core-shell nanostructure and untreated control showed no significant differences in percentage mean absorption between various treatments (Figure 2), indicating that the 


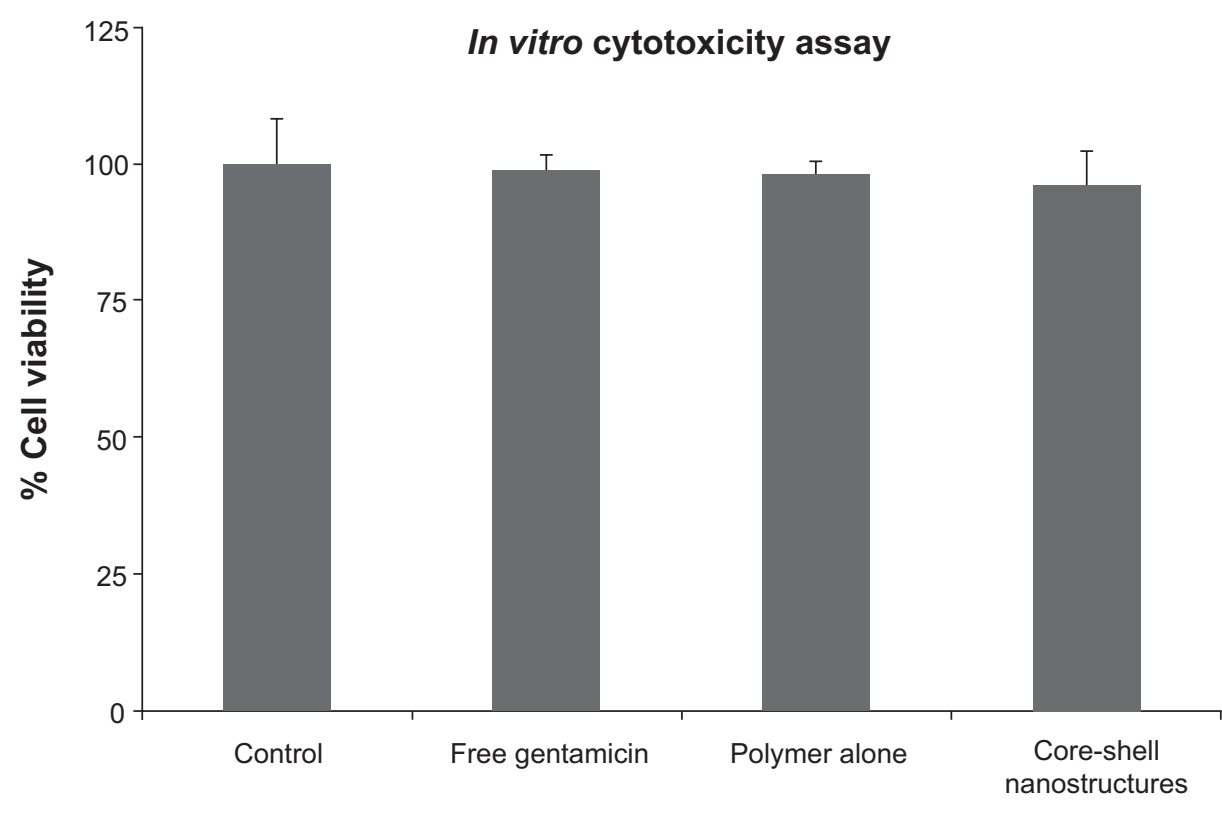

Treatment

Figure 2 MTS assay showing the percentage mean absorbance (depicted by \% cell viability at wavelength of $490 \mathrm{~nm}$ ) after incubating J774A.I cells with $250 \mu \mathrm{mL} \mathrm{L}^{-1}$ of core-shell nanostructures along with appropriate controls. Results are expressed as means \pm SD of 6 measurements. **P $<0.05$, ANOVA.

Notes: No significant difference between treatments at $P<0.05$.

polymers at doses similar to that of the free gentamicin or untreated control were non-toxic in vitro.

\section{In vivo toxicity assessment}

Based on degrees of inflammation and mineralization of kidney tissues, D2N had a median score of 0 (range 0 to 0.50 ), untreated control 0 (range 0 to 0.75 ) and $\mathrm{D} 2 \mathrm{G} 0.75$ (range 0 to 2.50 ), determined by compiling the individual data scored by the pathologists (Table 1). The Kruskal-Wallis statistical test showed that various treatments had a statistically significant effect on kidney scores $(P=0.0288)$. Moreover, Dunn's procedure for multiple comparisons showed that the median kidney score for D2G was significantly greater than the median kidney score for D2N $(P=0.0223)$. The other two comparisons (D2G vs the untreated control, and the untreated control vs D2N) did not differ significantly.

Table I Kidney scoring by individual pathologist

\begin{tabular}{|c|c|c|c|c|c|c|}
\hline \multirow[t]{2}{*}{ Kidney no. } & \multicolumn{2}{|c|}{ Kidney score - D2N } & \multicolumn{2}{|c|}{ Kidney score - untreated } & \multicolumn{2}{|c|}{ Kidney score - D2G } \\
\hline & $\begin{array}{l}\text { Ist } \\
\text { pathologist }\end{array}$ & $\begin{array}{l}\text { 2nd } \\
\text { pathologist }\end{array}$ & $\begin{array}{l}\text { Ist } \\
\text { pathologist }\end{array}$ & & $\begin{array}{l}\text { Ist } \\
\text { pathologist }\end{array}$ & $\begin{array}{l}\text { 2nd } \\
\text { pathologist }\end{array}$ \\
\hline I & 0 & 0 & 0 & 0 & $2 * * *$ & $2 * * *$ \\
\hline 2 & 0 & 0 & 0 & 0 & $2 * * *$ & $2 * * *$ \\
\hline 3 & 0 & 0 & I & 0 & I & I \\
\hline 4 & 0 & 0 & $0 *$ & $0 *$ & 0 & 0 \\
\hline 5 & 0 & 0 & $I^{*}$ & $0 *$ & 0 & $\mathrm{I}$ \\
\hline 6 & 0 & 0 & 0 & $0 *$ & $\mathrm{I} * *$ & I \\
\hline 7 & 0 & 0 & 0 & 0 & 0 & 0 \\
\hline 8 & 0 & 0 & 0 & 0 & $2 * * *$ & 0 \\
\hline 9 & I & 0 & 0 & 0 & 0 & 0 \\
\hline 10 & 0 & 0 & 0 & 0 & 0 & 0 \\
\hline
\end{tabular}

Notes: *Mineralization of renal epithelium lining renal papilla collecting ducts.

***Mineralized deposits randomly scattered within the cortex and in medulla.

***Indicates mineralized deposits in the cortex associated with inflammatory cells.

Kidney scores: 0 =normal; $I=$ minimal inflammation; $2=$ mild inflammation; $3=$ moderate inflammation; $4=$ marked inflammation

Abbreviations: D2G, mice group treated with $15 \mu \mathrm{g} \mathrm{g}{ }^{-1}$ body weight with free gentamicin; D2N, mice group treated with $15 \mu \mathrm{g} \mathrm{g}^{-1}$ body weight with core shell nanostructure. 
Histopathologically, the D2G-treated kidney tissues revealed minimal to mild lymphocytic inflammation associated with mineralized deposits (Figure 2). In contrast, the untreated or D2N tissues typically showed unremarkable to rare small peri-glomerular aggregates of mononuclear cells.

\section{In vivo efficacy}

The antibacterial activities of free gentamicin and the core shell nanostructures were compared in a $S$. typhimurium infection model in AJ 646 mice. The gentamicin nanostructures were tested at 2 different dosages, viz. 5 and $15 \mu \mathrm{g} \mathrm{g}^{-1}$ to evaluate the efficacy in in vivo bacterial clearance (Table 2). D1N resulted in significant reduction of viable bacteria both in the liver $\left(0.46 \log _{10}\right)$ and in the spleen $\left(0.25 \log _{10}\right)$ compared to the untreated control. However, D1G yielded significant reductions of viable bacteria in the liver $\left(0.48 \log _{10}\right)$ but not in the spleen relative to those recovered from the controls. In contrast, statistically significant reductions in the numbers of viable bacteria in the liver $\left(1.03 \log _{10}\right)$ and spleen $\left(0.29 \log _{10}\right)$ were observed for the $\mathrm{D} 2 \mathrm{~N}$ in comparison to the D2G and infected control $(P<0.05)$.

\section{Discussion}

Aminoglycosides are one of the most commonly used classes of antimicrobials against Gram-negative bacteria due to their high efficacy and low costs, but they have the potential to produce nephrotoxic and ototoxic side effects. ${ }^{19,20}$ Basically, aminoglycosides are low protein-binding drugs that can freely filter in the kidney glomeruli upon parenteral administration without metabolization in the body. However, around $10 \%$ of an administered aminoglycoside such as gentamicin can selectively accumulate in kidney tissue and may cause structural and functional damage to the renal tubular cells. Thus, development of new strategies aiming to prevent their accumulation in the renal tissues is highly desirable for reducing toxicity. Also, though aminoglycosides are highly efficacious in vitro, their in vivo activity is limited by an inability to traverse through the phagocytic cell membrane. This is because intracellular penetration of a drug molecule depends on its polarity, and polar drugs cannot permeate readily across nonpolar, lipophilic cell membranes. Hence, aminoglycosides such as gentamicin which are cationic and polar have relatively low permeabilities across cell membranes. ${ }^{18}$

One means to reduce toxicity and improve efficacy is to favor delivery of drugs to the infected organs. In the case of systemic infection with $S$. typhimurium, the target organs of infection are the spleen and liver. ${ }^{19}$ By encapsulating drug molecules in polymeric core-shell nanostructures where the physico-chemical characteristics of the shell have been designed to interact with lipid membranes, internalization by the tissue macrophage cells can be improved. This reduces transport of free gentamicin to renal tissues and enhances targeting to the liver or spleen. In this research, we utilized an amphiphilic Pluronic F-68 copolymer as the shell, and gentamicin was incorporated into the nanoparticle cores through cooperative electrostatic attractions. To fabricate the nanostructured complexes, a polyanionic $\mathrm{PAA}^{-+} \mathrm{Na}$ homopolymer ${ }^{10}$ and PAA ${ }^{-+} \mathrm{Na}-b$-PEO- $b$-PPO- $b$-PEO$b$-PAA ${ }^{-+} \mathrm{Na}$ block copolymer were co-dissolved and a polycationic gentamicin sulfate solution ${ }^{20}$ was added with sonication to form a final one-to-one ratio of anions to cations. In general, the polyacrylate components formed the core of the complex while the nonionic blocks extended outward into the aqueous medium to form the shells, so the polyacrylate homopolymer was used to "build up" the sizes and drug loadings of the cores as shown schematically in Figure 1. The non-ionic polyether shell afforded steric repulsion between particles and prevented macroscale agglomeration. The nanostructures prepared via this technique had a drug loading of $\sim 25 \%$ by weight of the polymer. However, while the $25 \%$ drug loading is high, it is noteworthy to observe that a significant amount of the charged drug was not incorporated and better understanding

Table 2 Efficacy cfu (log) reduction of free gentamicin or core-shell nanostructure polymers in liver and spleen from Salmonella-infected mouse

\begin{tabular}{lllll}
\hline Treatment & Liver cfu $(\log )(\mathrm{Cl})$ & Liver cfu $(\log )$ reduction & Spleen cfu $(\mathrm{log})(\mathrm{Cl})$ & Spleen cfu (log) reduction \\
\hline Infected control & $4.18(3.98,4.29)$ & 0.00 & $3.89(3.76,4.28)$ & 0.00 \\
DIN & $3.72(3.28,3.88)$ & $0.46^{*}$ & $3.64(3.50,3.69)$ & $0.25^{*}$ \\
DIG & $3.70(3.29,3.75)$ & $0.48^{*}$ & $3.98(3.83,4.01)$ & -0.09 \\
D2N & $3.1 I(2.99,3.53)$ & $1.07^{*}$ & $3.60(3.48,4.02)$ & $0.29^{*}$ \\
D2G & $3.95(3.92,3.98)$ & 0.23 & $4.23(4.13,4.32)$ & -0.34 \\
\hline
\end{tabular}

*Significantly different from control $(P<0.05)$.

Abbreviations: cfu, coloning forming units; $\mathrm{Cl}$, confidence interval; DIN, mice group treated with $5 \mu \mathrm{g} \mathrm{g}^{-1}$ body weight wiht core shell nanostructure; $\mathrm{D} 2 \mathrm{~N}$, mice group treated with $15 \mu \mathrm{g} \mathrm{g}^{-1}$ body weight with core shell nanostructure; DIG, mice group treated with $5 \mu \mathrm{g} \mathrm{g}^{-1}$ body weight with free gentamicin; D2G, mice group treated with I5 $\mu \mathrm{g} \mathrm{g}^{-1}$ body weight with free gentamicin. 
of this issue will be topic for continuing research. Also, gentamicin releases from the core-shell nanostructure could be a function of cooperativity between the homo and block copolymers and drug. This is evidenced from our previous findings wherein rate of release of gentamicin from nanostructure with pluronic F68 shell was relatively slow compared to free gentamicin at physiological $\mathrm{pH} 7.4$ and $37^{\circ} \mathrm{C} . .^{14,21}$ Even though the overall complexes had a significant net negative charge, they had small zeta potentials in DI water, ranging from $-0.7( \pm 0.2) \mathrm{mV}$. This suggests that the non-ionic PEO and PEO- $b$-PPO- $b$-PEO shells effectively screened the excess of anionic charges in the core from the environment. ${ }^{22}$ It has also been suggested that the rate of particle uptake by macrophage cells increases with an increase in size with the minimum recognizable size of $\sim 70$ to $85 \mathrm{~nm} .{ }^{23,24}$ In our experimental analysis, we observed that the size of the core-shell nanostructure was $\sim 210( \pm 17) \mathrm{nm}$ but a systematic study of the effect of size has not yet been conducted.

The core-shell nanostructures can be potentially realized in clinical situations by ruling out any toxicity associated with their use in biological systems. This was determined in vitro via an MTS assay which measures mitochondrial activity through the formation of a soluble formazan product which is directly proportional to the number of live cells in culture. ${ }^{25}$ MTS assays conducted upon incubation of cells with the coreshell nanostructures containing gentamicin showed no significant differences in absorption compared to untreated control, indicating that the polymers at doses similar to that of the free gentamicin or untreated control were non-toxic in vitro (Figure 2). Further, in vivo studies of renal histopathology were performed independently by two anatomic pathologists to screen for microscopic lesions. Each kidney was given a subjective score from 0 (unremarkable) to 4 (marked). Both evaluators noted minimal to mild increased inflammation in half of the kidneys in the mice group treated with free gentamicin. Additionally, both evaluators noticed mineral deposits within the renal cortex associated with inflammatory cells and these lesions were not present in kidneys from the untreated or core-shell nanostructure treated groups of mice. These lesions are consistent with either the deposition of a mineralized substance eliciting inflammation or chronic regions of inflammation with dystrophic mineralization. The inflammatory infiltrate in these kidneys were predominantly comprised of lymphocytes and macrophages, as has been similarly observed previously for gentamicin nephrotoxicity. ${ }^{26}$ However, the kidneys did not exhibit distended tubules, hyaline casts, tubular degeneration, glomerular basement membrane alterations or necrosis reported in gentamicin toxicity. ${ }^{27,28}$ Plausibly, the single dose administration of gentamicin at $15 \mu \mathrm{g} \mathrm{g}^{-1}$ body weight of mice may not have been enough to cause concentration dependent toxicity. Repeated administration for multiple times/days to the mice similar to previous studies may shed more light on the protective effects of gentamicin encapsulation against nephrotoxicty. ${ }^{27}$ Regardless, unremarkable to minimal renal lesions in untreated and core-shell nanostructure-treated mice suggest that gentamicin encapsulation may be protective in preventing adverse effects in the kidney.

Finally, AJ-646 mice were infected with $S$. typhimurium and treated with the core-shell nanostructures. The results indicated that at a $15 \mu \mathrm{g} \mathrm{g}^{-1}$ dose, free gentamicin was ineffective in bacterial clearance. In contrast Salmonella was
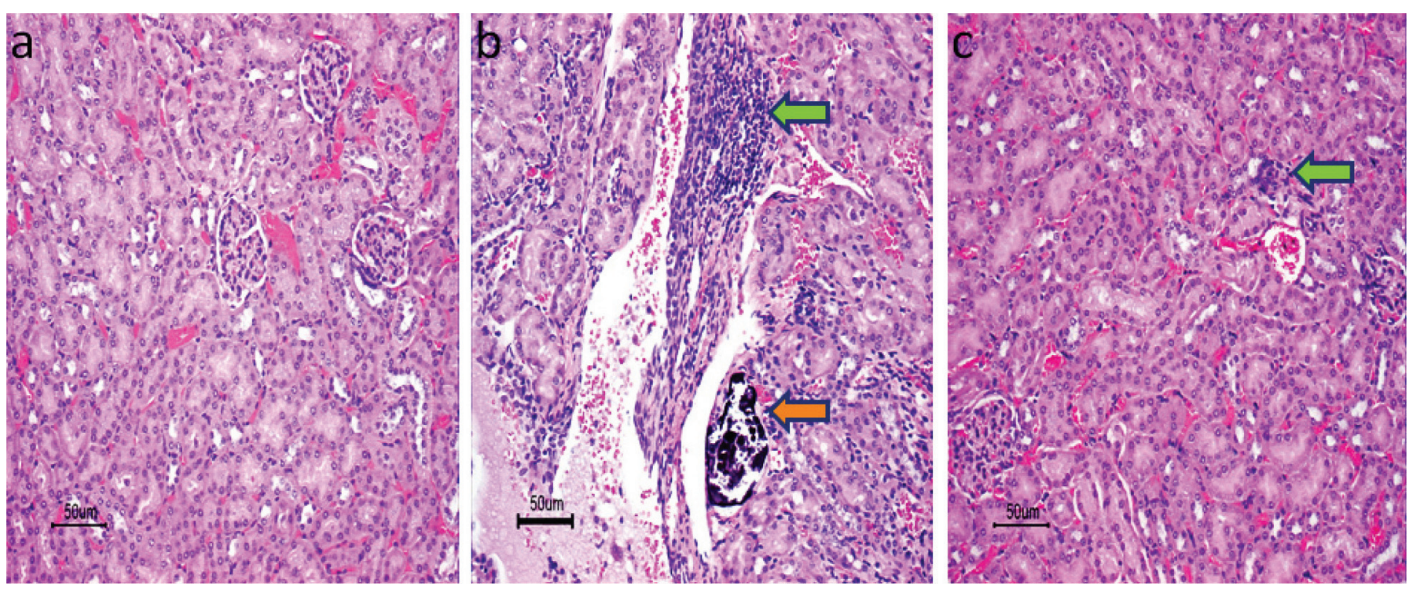

Figure 3 Histopathological microscopic images of kidney tissues of AJ-646 mice euthanized 5 days after intraperitoneal administration: A) Untreated control group; B) D2G-treated group: Minimal to mild lymphocytic inflammation (green arrow) associated with mineralized deposits (orange arrows); C) D2N-treated group: Typically unremarkable to rare small peri-glomerular aggregates of mononuclear cells (arrow). The tissues were hematoxylin and eosin stained. Scale bar represents $50 \mu \mathrm{m}$.

Abbreviations: D2G, mice group treated with $15 \mu \mathrm{g} \mathrm{g}^{-1}$ body weight with free gentamicin; D2N, mice group treated with $15 \mu \mathrm{g} \mathrm{g}^{-1}$ body weight with core shell nanostructure. 
reduced significantly in the liver and spleen tissues in the D2N treated group. Significant reductions in Salmonella were also observed on administration of multiple doses of D1N at $5 \mu \mathrm{g} \mathrm{g}^{-1}$ in the spleen and liver and in the liver of D1G-treated mice respectively. Previous studies conducted with liposomes in an in vivo Salmonella infection model have suggested that the gentamicin levels in plasma, liver and spleen are increased significantly upon encapsulation. ${ }^{29}$ This consequently has a favorable effect on the bacterial clearance as evidenced by $10^{4}$ reductions in the cfu counts of spleen and liver tissue. In addition, encapsulation increases the circulation time of the drug, thereby enhancing delivery to the target organ of infection and preventing the free drug from reaching the renal tissues. ${ }^{30}$ Furthermore, uptake studies in our laboratory have shown that increased amphiphilicity of the gentamicinpolymer core-shell nanostructure significantly improves the amount and rate of uptake by the macrophage cells. ${ }^{21}$ Thus, improved efficacy in Salmonella clearance by the D2N-treated mice could be an interplay of both enhanced delivery and increased uptake by the tissue macrophage cells.

Altogether, the current study shows that improved efficacy and delivery to the infected organs can be achieved by incorporating gentamicin in pluronic-based coreshell structures. This has a protective effect in preventing adverse toxic effects on the kidney. Future studies aiming to improve the intracellular targeting to the niche where the Salmonella reside may enhance the suitability of the coreshell antimicrobial DDS systems.

\section{Acknowledgments}

The authors are grateful to NSF DMR-0312046, Virginia Tech's Institute for Critical and Applied Sciences (ICTAS) and Department of Large Animal Clinical Sciences, Va- Md Regional College of Veterinary Medicine for funding.

\section{Disclosures}

The authors declare no conflicts of interest.

\section{References}

1. Kostarelos K. The emergence of nanomedicine: a field in the making. Nanomedicine. 2006;1:1-3.

2. Ahmad Z, Sharma S, Khuller GK. Chemotherapeutic evaluation of alginate nanoparticle-encapsulated azole antifungal and antitubercular drugs against murine tuberculosis. Nanomedicine. 2007;3:239-243.

3. Silva JG, Carvalho I. New insights into aminoglycoside antibiotics and derivatives. Curr Med Chem. 2007;14:1101-1119.

4. Ristuccia AM, Cunha BA. The aminoglycosides. Med Clin North Am. 1982;66:303-312.

5. Fattal E, Rojas J, Roblot-Treupel L, Andremont A, Couvreur P. Ampicillinloaded liposomes and nanoparticles: comparison of drug loading, drug release and in vitro antimicrobial activity. J Microencapsul. 1991;8:29-36.
6. Lutwyche P, Cordeiro C, Wiseman DJ, et al. Intracellular delivery and antibacterial activity of gentamicin encapsulated in $\mathrm{pH}$-sensitive liposomes. Antimicrob Agents Chemother. 1998;42:2511-2520.

7. Kabanov AV, Alakhov VY. Pluronic block copolymers in drug delivery: from micellar nanocontainers to biological response modifiers. Crit Rev Ther Drug Carrier Syst. 2002;19:1-72.

8. Kabanov AV, Batrakova EV, Alakhov VY. Pluronic block copolymers as novel polymer therapeutics for drug and gene delivery. J Control Release. 2002;82:189-212.

9. Batrakova EV, Kabanov AV. Pluronic block copolymers: evolution of drug delivery concept from inert nanocarriers to biological response modifiers. J Control Release. 2008;130:98-106.

10. Alakhov V, Klinski E, Lemieux P, Pietrzynski G, Kabanov A. Block copolymeric biotransport carriers as versatile vehicles for drug delivery. Expert Opin Biol Ther. 2001;1:583-602.

11. Neudeck BL, Alford TD, Faith NG, Czuprynski CJ. The poloxamer P85 is protective against Listeria monocytogenes invasion. Foodborne Pathog Dis. 2008;5:859-865.

12. Yang Z, Sahay G, Sriadibhatla S, Kabanov AV. Amphiphilic block copolymers enhance cellular uptake and nuclear entry of polyplex-delivered DNA. Bioconjug Chem. 2008;19:1987-1994.

13. Tian Y, Bromberg L, Lin SN, Hatton TA, Tam KC. Complexation and release of doxorubicin from its complexes with pluronic P85-bpoly(acrylic acid) block copolymers. J Control Release. 2007;121: 137-145.

14. Ranjan A, Seleem M, Jain J, Sriranganathan N, Riffle JS, Kasimanickam R. Drug delivery using novel nanoplexes against a Salmonella mouse infection model. J Nanopart Res. 2009:DOI 10.1007/ s11051-009-9641-y.

15. Bajema IM, Hagen EC, Hansen BE, et al. The renal histopathology in systemic vasculitis: an international survey study of inter- and intraobserver agreement. Nephrol Dial Transplant. 1996;11:1989-1995.

16. Joss N, Morris S, Young B, Geddes C. Granulomatous interstitial nephritis. Clin J Am Soc Nephrol. 2007;2:222-230.

17. Tynes VV. Drug therapy in pet rodents. Vet Med. 1998;93:988-991.

18. Lecaroz C, Gamazo C, Blanco-Prieto MJ. Nanocarriers with gentamicin to treat intracellular pathogens. J Nanosci Nanotechnol. 2006;6: 3296-3302.

19. Lahiri A, Das P, Chakravortty D. Salmonella typhimurium: Insight into the multi-faceted role of the LysR-type transcriptional regulators in Salmonella. Int J Biochem Cell Biol. 2009.

20. Abraham AM, Walubo A. The effect of surface charge on the disposition of liposome-encapsulated gentamicin to the rat liver, brain, lungs and kidneys after intraperitoneal administration. Int J Antimicrob Agents. 2005;25:392-397.

21. Ranjan A, Pothayee N, Seleem MN, Sriranganathan N, Riffle JS, Kasimanickam R, et al. In vitro trafficking and efficacy of coreshell nanostructures for treating intracellular Salmonella. 2009:53.

22. Tang GP, Zeng JM, Gao SJ, et al. Polyethylene glycol modified polyethylenimine for improved CNS gene transfer: effects of PEGylation extent. Biomaterials. 2003;24:2351-2362.

23. Chono S, Morimoto K. Uptake of dexamethasone incorporated into liposomes by macrophages and foam cells and its inhibitory effect on cellular cholesterol ester accumulation. J Pharm Pharmacol. 2006;58:1219-1225.

24. Rudt S, Muller RH. In vitro phagocytosis assay of nano- and microparticles by chemiluminescence. III. Uptake of differently sized surface-modified particles, and its correlation to particle properties and in vivo distribution. Eur J Pharm Sci. 1993;1:31-39.

25. Theas MS, Rival C, Jarazo-Dietrich S, Jacobo P, Guazzone VA, Lustig L. Tumour necrosis factor-alpha released by testicular macrophages induces apoptosis of germ cells in autoimmune orchitis. Hum Reprod. 2008;3:1865-1872.

26. Bledsoe G, Crickman S, Mao J, et al. Kallikrein/kinin protects against gentamicin-induced nephrotoxicity by inhibition of inflammation and apoptosis. Nephrol Dial Transplant. 2006;21:624-633. 
27. Stojiljkovic N, Veljkovic S, Mihailovic D, et al. Protective effects of pentoxifylline treatment on gentamicin-induced nephrotoxicity in rats. Ren Fail. 2009;31:54-61.

28. Ulutas B, Sarierler M, Bayramli G, Ocal K. Macroscopic findings of idiopathic congenital megaoesophagus in a calf. Vet Rec. 2006;158:26.

29. Cordeiro C, Wiseman DJ, Lutwyche P, et al. Antibacterial efficacy of gentamicin encapsulated in $\mathrm{pH}$-sensitive liposomes against an in vivo Salmonella enterica serovar typhimurium intracellular infection model. Antimicrob Agents Chemother. 2000;44:533-539.
30. Webb MS, Boman NL, Wiseman DJ, et al. Antibacterial efficacy against an in vivo Salmonella typhimurium infection model and pharmacokinetics of a liposomal ciprofloxacin formulation. Antimicrob Agents Chemother. 1998;42:45-52.

International Journal of Nanomedicine

\section{Publish your work in this journal}

The International Journal of Nanomedicine is an international, peerreviewed journal focusing on the application of nanotechnology in diagnostics, therapeutics, and drug delivery systems throughout the biomedical field. This journal is indexed on PubMed Central, MedLine, CAS, SciSearch $\AA$, Current Contents ${ } /$ Clinical Medicine,
Journal Citation Reports/Science Edition, EMBase, Scopus and the Elsevier Bibliographic databases. The manuscript management system is completely online and includes a very quick and fair peer-review system, which is all easy to use. Visit http://www.dovepress.com/ testimonials.php to read real quotes from published authors.

Submit your manuscript here: http://www.dovepress.com/international-journal-of-nanomedicine-journal 